\title{
Factores legales que impiden una articulación de la provincia de La Unión con el desarrollo de la región Arequipa*
}

Legal factors that imply an articulation of the province of La Unión with the development of the Arequipa region

Gregorio Nicolás Cusihuamán Sisa**

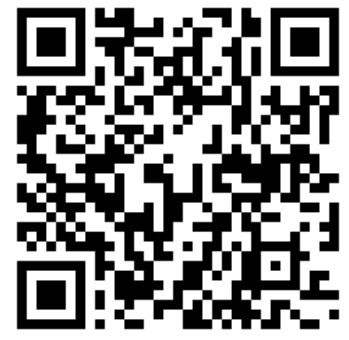

\section{Resumen}

La región Arequipa en los últimos dos quinquenios ha crecido consistentemente en un $6,64 \%$ en su PBI anual, razón por la cual se ubica entre las tres regiones con mayor índice de competitividad regional a nivel nacional; sin embargo, La Unión es una de sus ocho provincias cuya desarticulación es notoria, su Índice de Desarrollo Humano (IDH) está por debajo del 0,30 mostrando gran desigualdad y una clara desconexión. El objetivo es identificar los factores legales que desarticulan la provincia de La Unión con el desarrollo de la región Arequipa, lo que atenta contra los Derechos Humanos de sus pobladores y genera discriminación además de impedir el desarrollo de la provincia. En cuanto a la metodología, se trata de una investigación aplicada del tipo mixta (cuali-cuantitativa), descriptiva con carácter analítica no experimental. Tiene carácter interdisciplinario pues se desarrolla desde diferentes perspectivas, las técnicas utilizadas son la encuesta, la entrevista y la observación. Los resultados muestran que los factores determinantes que desarticulan a la provincia de La Unión, son dispositivos legales

\footnotetext{
* Artículo original derivado del Proyecto "Desarrollo educativo de Arequipa" Financiado por Universidad Nacional de San Agustín de Arequipa Perú entre agosto 2018 y mayo 2019.

** Docente universitario, Universidad Nacional de San Agustín de Arequipa Perú. Arequipa, Perú. E-mail: gcusihuaman@unsa.edu.pe. ORCID: http://orcid.org/0000-0002-0568-8065.
}

\author{
Sinergias educativas \\ Enero - junio Vol. 5 -1 - 2020 \\ http://sinergiaseducativas.mx/index.php/revista/ \\ elSSN: 2662-6661 \\ revistasinergia@soyuo.mx \\ Recepción: 1 mayo 2018 \\ Aprobación: 19 junio 2019 \\ Pag 288 - 306 \\ Disponible en \\ http://www.redalyc.org/articulo.oa?id=57356143200 \\ 22 \\ Atribución/Reconocimiento-NoComercial- \\ Compartirlgual 4.0 Licencia Pública Internacional - \\ CC BY-NC-SA 4.0 \\ https://creativecommons.org/licenses/by-nc- \\ sa/4.0/legalcode.es
}


referidos a la distribución de recursos económicos, con el agravante de que la provincia, en el año 2005 mediante el decreto $\mathrm{N}^{\circ}$ 05-2005, fue declarada Área Natural Protegida, norma que impide explotar de manera formal los recursos mineros en la provincia.

Palabras clave: desarrollo rural, Discriminación legal, desarticulación, Derechos Humanos, La Unión.

\section{Abstract}

The Arequipa region in the last two five year has grown consistently by $6.64 \%$ in its annual GDP, which is why it ranks among the three regions with the highest regional competitiveness index at the national level; However, La Unión is one of its eight provinces whose dismantling is notorious, its Human Development Index (HDI) is below 0.30, showing great inequality and a clear disconnection. The objective is to identify the legal factors that disrupt the province of La Unión with the development of the Arequipa region, which undermines the human rights of its inhabitants and generates discrimination in addition to preventing the development of the province. As for the methodology, it is an applied research of the mixed type (qualitative-quantitative), descriptive with non-experimental analytical character. It is interdisciplinary in nature since it is developed from different perspectives, the techniques used are the survey, the interview and the observation. The results show that the determining factors that dismantle the province of La Unión, are legal devices referred to the distribution of economic resources, with the aggravating factor that the province, in 2005 by decree No. 05-2005, was declared Protected Natural Area, a rule that prevents the mining resources in the province from being formally exploited.

Key words: rural development, Legal discrimination, disarticulation, Human Rights, The Union.

\section{Introducción}

La investigación analiza una de las ocho provincias de Arequipa, la provincia de La Unión, porque muestra una clara desarticulación con las siete restantes, su nivel de pobreza es severo, por encima del $50 \%$ 
de su población, y un índice de desarrollo humano inferior al 0,30, considerando que Arequipa región se ubica como la segunda región competitiva del Perú, de acuerdo al Índice de Competitividad Regional - INCORE, (Instituto Peruano de Economía, 2018). Este análisis toma en consideración cinco factores como son educación, salud, desarrollo social (pobreza), infraestructura y modernidad. Respecto al Índice de Desarrollo Humano-IDH (BCR, 2016), a nivel nacional Arequipa presenta un indicador de 0.60 , promedio superior a la media nacional. Sin embargo, la provincia de La Unión muestra un 0.29, siendo el distrito de Tauría con el nivel más bajo de IDH con 0.14 (PNUD, 2012) es decir, un bajo nivel de calidad de vida. Esta evaluación contrasta con la información publicada por el Consorcio de Investigación Económico y Social (CIES) donde se señala que La Unión tiene un $50 \%$ de pobreza (Zegarra López, 2015). En la presente investigación proponemos siete ejes de desarrollo que se podrían articular para generar sinergias de desarrollo. A su vez, consideramos que el sector agricultura sería fundamental para lograr un crecimiento económico sostenido que a la postre se convierta en desarrollo, siempre y cuando se contemplen tres aspectos: sostenibilidad financiera, permitiendo márgenes de ganancia apropiados y el pago de jornales adecuados. Sostenibilidad ecológica, es decir, prácticas que no dañen el medio ambiente tanto por el uso de agroquímicos como por la reducción de fertilizantes. Y la sostenibilidad genética, favoreciendo la diversidad genética necesaria para enfrentar cambios ambientales impredecibles (Consorcio de Universidades, 2016). Sin embargo, para sostener estos tres aspectos es necesario una base económica con respaldo legal que no existe actualmente en esta provincia.

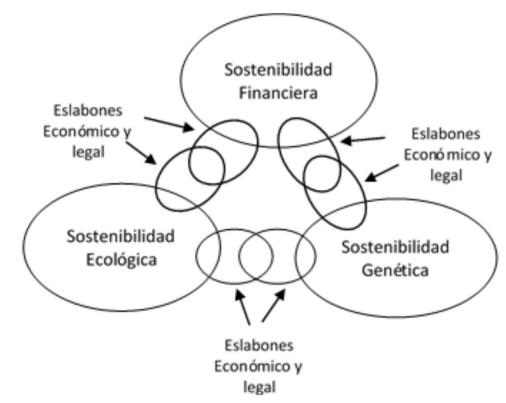

Figura $\mathbf{N}^{\circ} 01$

Elaboración propia con información de (Consorcio de Universidades, 2016) 
Para nuestro análisis consideramos la propuesta analítica de Perroux quien define el desarrollo como "la combinación de los cambios mentales y sociales de una población que la vuelven apta para crecer, cumulativa y durablemente su producto real global". Agrega el autor en un comentario que "la consecuencia que no haya desarrollo económico es la falta de crecimiento económico, social y cultural" (Guillen Romo, 2005, pág. 15). Los elementos desarticuladores no se limitan estrictamente a la infraestructura comunicacional y de transportes, sino también a la diversidad de etnias con usos y costumbres propios. La propuesta analítica respecto a la articulación se basa en lo señalado por Rimisp, quien afirma que la articulación es el proceso mediante el cual se genera sinergia entre las acciones y los recursos de los diversos involucrados en un campo concreto de gestión pública (RIMISP, 2018).

Se considera que el sector educación en las condiciones precarias actuales no coadyuva como elemento articulador. A nivel nacional, la estadística señala dos aspectos como factores excluyentes: el sector rural y el segmento femenino. Siempre hay mayor analfabetismo en el área rural y, a su vez, las mujeres tienen un mayor número de analfabetos que los hombres, destacando esta cifra en la provincia de La Unión.

\begin{tabular}{l|l|l}
\multicolumn{3}{c}{ Tasa de analfabetismo en Perú 2017} \\
\hline & Urbano & Rural \\
\hline Hombres & 5.3 & 7.4 \\
\hline Mujeres & 1.7 & 23.5 \\
\hline
\end{tabular}

Fuente: (ESCALE, 2016)

Tasa de analfabetismo en la provincia de La Unión 2017

\begin{tabular}{l|l}
\hline & Rural \\
\hline Hombres & 8.6 \\
\hline Mujeres & 32.6 \\
\hline
\end{tabular}

(ESCALE, 2016) 
Por tanto, no resulta una mera coincidencia, por el contrario, es el reflejo de una tradicional actitud respecto a ambos temas: es evidente la discriminación étnica, así como la discriminación por género. En el primer caso, como señala Santos en la revista Debates, "la discriminación implica prácticas que niegan o limitan derechos y oportunidades a determinados grupos pero no a otros, en consecuencia, hay un trato diferenciado" (Santos, 2014, pág. 7) que inicialmente se presenta como una práctica común, de tipo cultural, y que todos la aceptan, tanto hombres como mujeres, en el sector urbano como en el sector rural. Dentro de este análisis, se hace notar el proceso de retroalimentación que genera la discriminación y la exclusión social.

Es difícil definir con precisión lo que significa realmente el desarrollo rural. Hay distintas percepciones y cada cual tiene su propia idea de desarrollo. A su vez, el término progreso ha tenido una larga y sinuosa interpretación, a ello se suma el concepto de modernidad que tiene fundamentos vinculados al desarrollo social por lo que resulta necesario comprender su propósito en conjunto. Como lo reconoce Alain Touraine, "la modernización indica la capacidad que tiene un sistema social de producir modernidad; el desarrollo se refiere a la voluntad que tienen los actores sociales o, mejor aún, políticos de transformar la sociedad" (Touraine, 1995, p. 10). Debemos reconocer que diversos tratados analizan el desarrollo desde una perspectiva eminentemente urbana, desde ahí podríamos afirmar que esa modernidad y posmodernidad tienen esencia social urbana. Tenemos a Chigbu quien afirma que "el concepto de urbanización evoca una atención abrumadora o urgencia de una acción global, pero la ruralización no atrae ni plantea tal atención para la acción de masas" (Chigbu, 2015, p. 1068).

En el artículo consideramos como sector rural la zona en donde las prácticas agrícolas son preminentes en su sistema productivo (Matijasevic Arcila \& Ruiz Silva, 2013), al margen que en información oficial se considere urbana y rural.

En este análisis se hace necesario replantear la perspectiva del modelo de desarrollo rural. Investigadores de la Pontificia Universidad Católica del Perú (PUCP) inician el trabajo de desarrollo humano, migraciones y capital social señalando la 
importancia de la propuesta basada en el enfoque de capacidades de Amartya Sen, congruente con las libertades reales individuales en un contexto de desarrollo colectivo (Ansion, Mujica, Piras, \& Villacorta, 2013). Una alternativa viable que confirma el deseo natural de libertad en un contexto de justicia y que coincide con Sen cuando afirma: "Mientras que los tigres están protegidos, no hay nada que proteja a los miserables seres humanos que tratan de ganarse la vida trabajando en ese bosque profundo, bello y peligroso" (Sen, 2013, p. 183).

Por otro lado, identificamos el criterio de desarrollo de Daza quien afirma que:

"La modernidad se basa en la esperanza de progreso material indefinido, pero la modernidad es algo más: se encuentra conectada con la superación de aquel viejo régimen caracterizado por la irracionalidad en la vida social, el Estado absoluto, los privilegios individualistas discriminatorios, las diferencias culturales y comunitarias convertidas en una tradición que se opone a la posibilidad del cambio social y por la ausencia de unidad en lo diverso y de sociedades cohesionadas por intereses universales" (Daza, 2010, p. 79).

En alusión a la innovación como elemento principal al proceso de desarrollo anhelado, Berdagué, en un artículo publicado en "Economía Informa", señala que la innovación en la agricultura se puede definir como un proceso de aprendizaje social con múltiples actores involucrados que genera $y$ pone en uso nuevo conoci $\neg$ miento, y que expande las capacidades y las oportuni $\neg$ dades de los pobres (Amaro-Rosales \& De Gortari Rabicla, 2016). Otro hecho destacable lo describe la revista "Economic Botanic", donde se manifiesta que la agricultura en la zona rural es progresivamente responsabilidad de las personas mayores, ya que la mayoría de los jóvenes migran a las ciudades, exactamente como se aprecia en la provincia de La Unión, por lo cual la agricultura está dejando de ser una actividad familiar.

Nuestra aproximación está por el lado de Sen, quien concibe al desarrollo como un proceso integrado de expansión de libertades fundamentales relacionadas entre sí, asumiendo lo que dice este 
reconocido economista en otros análisis referidos a la conexión de la razón de la evaluación y la razón de la eficacia, ya que considera a ambas como elementos concomitantes a la libertad del individuo en el desarrollo social (Sen, 2013).

Otro aspecto relevante es la discriminación racial, asumido en la investigación, y como señala Marisol Angles (tomado de Naciones Unidas, Ginebra 1989), "la discriminación racial que viven comunidades y pueblos originarios es resultado de un largo proceso histórico de conquista, penetración y marginación, que ha ido acompañado de actitudes de superioridad y de una concepción del indígena como ser "primitivo" e "inferior"” (Angles Hernandez, 2014, pág. 263). Si bien la demostración se expresa en términos estrictamente legales y cómo estos inciden en la economía de los sectores rurales de Arequipa, se hace necesario reconocer que la discriminación sistémica existe, como señala Angles, es omnipresente y está fuertemente arraigada en el comportamiento y la organización de la sociedad y a menudo implica actos de discriminación indirecta o no cuestionada contra algunos grupos y que puede consistir en normas legales, políticas, prácticas o actitudes culturales predominantes en el sector público o privado (Angles, $\mathrm{p}$. 264).

\section{Materiales y métodos}

Se trata de una investigación descriptiva con carácter analítica no experimental transversal, cuyos fundamentos teóricos nos permiten adaptarnos a las circunstancias rurales. En un primer nivel del trabajo de campo, identificamos los once distritos de la provincia con deficiente acceso vial, índices de desarrollo muy por debajo del promedio, por lo que se propone levantar información de base. La provincia tiene una población de 14763 según el INEI (MINSA, 2016). La estructura poblacional muestra que el $29 \%$ está entre los 0 y 12 años, el $15 \%$ entre 13 y 25 años, el $40 \%$ entre 25 y 60 años y el $15 \%$ entre 60 y más años. Otro aspecto relevante es la proyección histórica de una disminución demográfica al margen que La Unión haya sido considerada como Área Natural Protegida de acuerdo al DS No 027-2005-AG, cuyo propósito es de cautelar el mantenimiento de la diversidad biológica. Desde el punto de vista 
lingüístico el $36 \%$ solo habla el quechua, el $46 \%$ castellano, el $3 \%$ aimara y el $15 \%$ el quechua y el castellano. Con estas premisas se planteó una distribución muestral proporcional a la población de cada distrito, se realizaron un total de 408 encuestas. A su vez se realizaron entrevistas, tanto a líderes comunales como a autoridades locales. Se aplicó una guía de observación debido a que las personas del lugar tienen aversión al trato con personas desconocidas por lo que se contrató a un guía del lugar, tanto para el acercamiento con los pobladores como para las citas con los líderes y autoridades. El proceso metodológico contempla la contrastación de los resultados obtenidos con información oficial de instituciones públicas y fuentes reconocidas.

\section{Resultados}

En primer aspecto revisamos la discriminación sistemática en la provincia de La Unión, la que se expresa en tres niveles de manera directa, generando externalidades en cada nivel de discriminación.

El primer nivel se presenta el 23 de mayo de 2005. Mediante Decreto Supremo No27-2005-AG, se establece la "Reserva Paisajística Subcuenca del Cotahuasi" como un área protegida en el Perú con una extensión de 490550 hectáreas y con una serie de ventajas para el ecosistema, las limitaciones están descritas en la Ley $\mathrm{N}^{\circ} 26834$ de áreas naturales protegidas, publicada el 17 de junio de 1997, donde en su artículo $\mathrm{N}^{\circ} 21$ dice: "De acuerdo a su naturaleza y objetivos, las Áreas Naturales Protegidas (ANP) cumplen una serie de limitaciones, principalmente en la extracción natural de recursos, contemplando una gradualidad, en otros términos limita la actividad minera e industrial, fortaleciendo la conservación del medio ambiente" (Congreso de la República, 1997). Esta disposición favorece la conservación de las ANP, sin embargo, no hay ninguna compensación económica a la población que habita en la provincia, generando el despoblamiento y evidenciando una discriminación directa a los pobladores de La Unión.

El segundo nivel de discriminación se presenta en la distribución de los recursos en las municipalidades del país mediante el Decreto Legislativo 776; por un lado, se regula la captación de tributos de los agentes económicos $\mathrm{y}$, por el otro, se exonera de pagos a 
comunidades campesinas, a universidades y a concesiones mineras, entre otros. Hablando estrictamente de la provincia en estudio queda claro que los ingresos por tributos son escasos por tratarse de un ANP y parte de sus habitantes son comunidades campesinas, por lo que están exonerados del tributo. Con esta premisa reconocemos lo señalado en la norma donde establece que la asignación mensual del Fondo de Compensación Municipal (Foncomún) estará directamente relacionada con el desempeño de la recaudación fiscal del mes anterior; cuando la recaudación es buena, la asignación del mes siguiente crece y cuando la recaudación se reduce, los municipios reciben una menor asignación (Ministerio de Economía y Finanzas, Portal de transparencia económica, 2004). Este segundo nivel muestra claramente que la provincia queda restringida de ingresos por Foncomún, al margen de que el índice de ruralidad favorecería, pero la evidencia la demostramos en la tabla comparativa de gastos de capital al año fiscal 2017, no se consideró gastos corrientes.

Tabla $\mathrm{N}^{\circ} 01$

Tabla comparativa de distribución de recursos año fiscal 2017 del Foncomún (En nuevos soles, solo se considera gastos de capital)

\begin{tabular}{|c|c|c|c|}
\hline \multicolumn{2}{|c|}{ Provincia de La Unión } & \multicolumn{2}{|l|}{ Provincia de Arequipa } \\
\hline Prov. La Unión & $10706418^{*}$ & Prov.Arequipa & $540832697^{*}$ \\
\hline Dist. Alca & 521423 & Dist. ASA & 21967752 \\
\hline Dist. Charcana & 106634 & Dist. Cayma & 10702276 \\
\hline Dist. Huaynacotas & 592313 & $\begin{array}{ll}\text { Dist. } & \text { Cerro } \\
\text { Colorado } & \end{array}$ & 29469195 \\
\hline Dist. Pampamarca & 279599 & Dist. Hunter & 2894156 \\
\hline Dist. Puyca & 709667 & $\begin{array}{l}\text { Dist. } \quad \text { Mariano } \\
\text { Melgar }\end{array}$ & 11356806 \\
\hline Dist. Quechualla & 72620 & Dist. Miraflores & 4723009 \\
\hline Dist. Sayla & 185121 & Dist. Paucarpata & 13480832 \\
\hline Dist. Tauria & 135185 & Dist. Sachaca & 5040861 \\
\hline Dist. Tomepampa & 100206 & Dist. Socabaya & 11178931 \\
\hline Dist. Toro & 168511 & Dist. Tiabaya & 13754878 \\
\hline & $\begin{array}{l}\text { Ministerio c } \\
\text { DMUN por } \\
\text { lesto, gasto c }\end{array}$ & $\begin{array}{l}\text { inanzas, Presupue } \\
\text { ncias, 2017) * } \\
\text { al }\end{array}$ & $\begin{array}{l}\text { stos } 2017 \\
\text { todo el }\end{array}$ \\
\hline
\end{tabular}

Es notoria la diferencia. Se debe reconocer que en la provincia de $\mathrm{La}$ Unión se tiene un índice de pobreza total del 57,60\% y una pobreza extrema de 26,80\% (PCM, 2012), con todo lo que implica ello 
respecto a la ausencia de servicios básicos como luz, agua, desagüe, etc. En algunos casos extremos como el distrito de Quechualla cuya capital es Velinga, que a la fecha no tiene acceso vial, la única forma de llegar es a través de un camino de herradura y sus alimentos los trasladan en animales de carga.

El tercer nivel de discriminación legal lo percibimos de manera más elocuente en la medida en que si consideramos que La Unión es ANP no goza de canon minero, cuya distribución está dada en base a la cantidad de habitantes, por lo que se configura en un círculo vicioso de discriminación legal. En calidad de área natural protegida, los pobladores no pagan tributos o son muy escasos, no hay industria ni minería, tampoco hay fondo de compensación especial por ser ANP, por tanto, su economía es estática; por otro lado, el canon se distribuye en base a la cantidad de habitantes, como la provincia experimenta una masiva migración debido a que no se dinamiza la economía disminuye la cantidad de habitantes por ende, las inversiones del gobierno regional decaen.

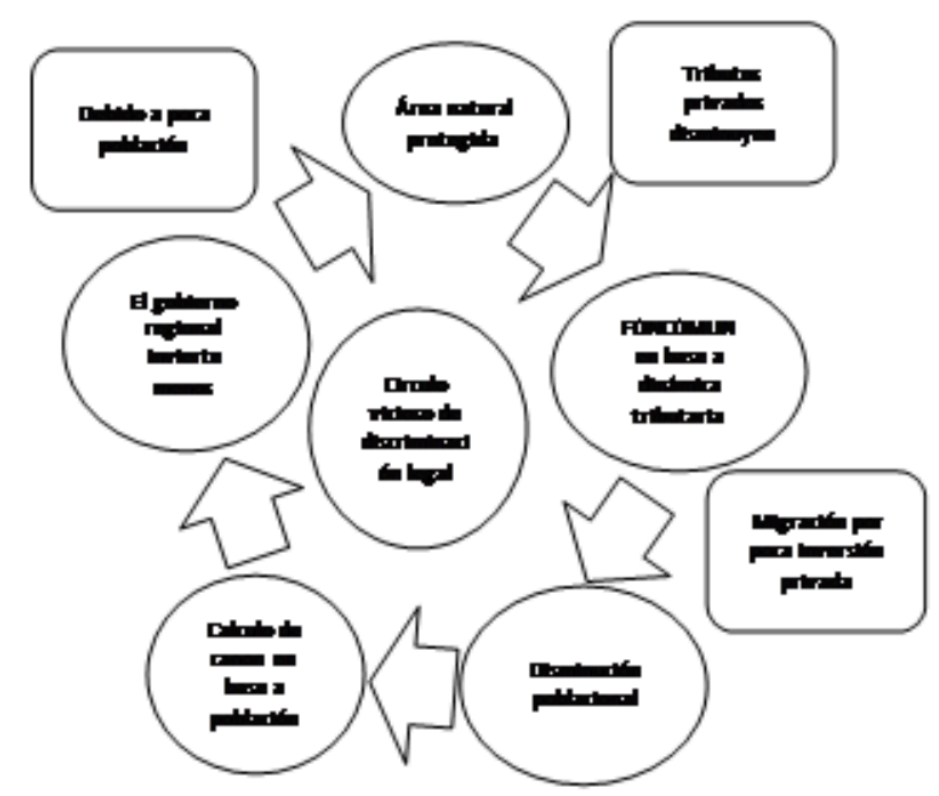

Figura $\mathbf{N}^{\circ} 02$

Círculo vicioso de la discriminación legal Propia

Queda demostrado que la declaración de Área Natural Protegida de la reserva paisajística de la subcuenca del Cotahuasi en el 2005 perjudicó al desarrollo de la provincia en la medida que no se generó 
un fondo de compensación especial, sabiendo que la dinámica del Foncomún está basada en el pago de tributos, por otro lado, las limitaciones a inversiones mineras y de industria debilitó los ingresos. Así como, los ingresos por canon de acuerdo a la Ley $\mathrm{N}^{\circ}$ 27506 serán distribuidos tomando en cuenta prioritariamente la cantidad de habitantes; la provincia con escasos recursos ha experimentado una migración que impide se realicen inversiones importantes, generando un ANP despoblada.

En la tabla $\mathrm{N}^{\circ} 2$ se muestra que sus recursos son largamente limitados respecto a canon minero, considerando que el sistema de distribución de recursos está limitado por ley como mencionamos anteriormente.

\section{Tabla $\mathbf{N}^{\circ} 2$}

Criterios de distribución de canon según ley № 27506

\begin{tabular}{|c|c|c|}
\hline$\%$ & Beneficiarios & Criterios \\
\hline $10 \%$ & $\begin{array}{l}\text { Municipios distritales donde se } \\
\text { exploten los recursos }\end{array}$ & $\begin{array}{l}\text { Si existen más de una } \\
\text { municipalidad en partes } \\
\text { iguales }\end{array}$ \\
\hline $25 \%$ & $\begin{array}{l}\text { Municipios de la provincia donde se } \\
\text { exploten los recursos naturales }\end{array}$ & $\begin{array}{l}\text { Según población y } \\
\text { necesidades básica } \\
\text { insatisfechas }\end{array}$ \\
\hline $40 \%$ & $\begin{array}{l}\text { Municipios del departamento donde } \\
\text { se exploten los recursos naturales }\end{array}$ & $\begin{array}{l}\text { Según población y } \\
\text { necesidades básica } \\
\text { insatisfechas }\end{array}$ \\
\hline \multirow{2}{*}{$25 \%$} & \multirow{2}{*}{ Gobierno regional } & $80 \%$ Gobierno regional \\
\hline & & Universidad \\
\hline
\end{tabular}

A partir de la ley $\mathrm{N}^{\circ} 27506$ el canon se debe distribuir en función de la cantidad poblacional y las necesidades insatisfechas, sin embargo solo se toma en cuenta la población; por ello, se considera que el 
sector rural como son los distritos de la provincia de La Unión son discriminados tal como se constató en la tabla $\mathrm{N}^{\circ} 3$.

\section{Tabla $\mathbf{N}^{\circ} 3$}

Tabla comparativa de distribución de recursos por canon minero 2017 (En nuevos soles)

\begin{tabular}{|c|c|c|c|}
\hline \multicolumn{2}{|c|}{ Provincia de La Unión s/2 210022} & \multicolumn{2}{|c|}{ Provincia de Arequipa s/142 334339} \\
\hline La Unión & 329576 & Arequipa & 2034974 \\
\hline Alca & 371545 & ASA & 6447170 \\
\hline Charcana & 75906 & Cayma & 7933457 \\
\hline Huaynacotas & 325716 & Cerro Colorado & 28387524 \\
\hline Pampamarca & 216271 & Hunter & 3349558 \\
\hline Puyca & 514123 & Mariano Melgar & 4880569 \\
\hline Quechualla & 35974 & Miraflores & 3382454 \\
\hline Sayla & 105041 & Paucarpata & 9869177 \\
\hline Tauria & 59380 & Sachaca & 3890903 \\
\hline Tomepampa & 57613 & Socabaya & 8468286 \\
\hline Toro & 118968 & Tiabaya & 10791899 \\
\hline
\end{tabular}

SNMPE (Sociedad Nacional de Minería, Petróleo y Energía, 2017)

La tabla $\mathrm{N}^{\circ} 3$ muestra una brecha amplia entre los gastos presupuestados por los distritos de La Unión con los distritos de Arequipa, no hay ningún distrito de La Unión que alcance un ingreso con capacidad para inversiones en infraestructura (en ningún caso supera siquiera el medio millón, sin embargo en Arequipa todos superan los dos millones), menos aún en el distrito que hacemos mención, Quechualla, que solo tiene un ingreso de 35974 soles, lo que no permite ejecutar el asfaltado del acceso vial por su propia cuenta, salvo si el gobierno regional tiene la voluntad de invertir en esta obra, en tal sentido la discriminación legal queda probada que impide ingresos mayores.

\section{Discusión}

Se considera que las restricciones legales en la distribución de recursos impiden una mejor calidad de vida de los pobladores de la Provincia de La Unión, generando así un atentado a los Derechos 
Humanos de los miembros de esta comunidad. En un análisis panorámico de los Derechos Humanos desde la época de la Independencia, Guadarrama señala que la perspectiva humanística se articula primero con las exigencias de las circunstancias políticas y sociales de esa época, las cuales comenzaban a demandar no sólo independencia política sino también respeto por los Derechos Humanos, en especial de los indígenas y esclavos africanos. Así como mayor justicia social para campesinos, artesanos, criollos y otros sectores marginados o discriminados, en especial las mujeres, en tal sentido ha estado orgánicamente imbricada de algún modo con la filosofía política del pensamiento universal (Guadarrama Gonzales, 2015). Al margen que sea tradición histórica, los Derechos Humanos siempre han sido una constante incompleta en el desarrollo de los pueblos latinoamericanos. Serrano y Vázquez señalan:

"Que son derechos subjetivos, expectativas que se forman las personas en relación con la acción u omisión de los estados, las empresas, los poderes fácticos y del resto de las personas respecto a ciertos bienes primarios constitutivos de lo que se considera la dignidad humana" (Serrano \& Vázquez, 2011, p. 10)

A su vez, los autores establecen diferencias entre el derecho subjetivo, los derechos naturales y los derechos universales: los primeros están referidos a los derechos inherentes al ser humano en el momento de realizar transacciones. Lo segundo radica en que son indispensables e inalienables, su respaldo es la ley, por lo que concebimos al derecho humano más que como respaldo natural, como forma de vivir ciudadanía.

En la concepción de los Derechos Humanos es ineludible soslayar la justicia, Gustavo Gutiérrez decía: "Ay del que edifica su casa sin justicia y sus pisos sin derecho" (1980), y es que la justicia trae de la mano una serie de connotaciones como la justicia social, la discriminación económica, étnica, cultural, entre otras. Es relevante ver la trayectoria histórica de la Iglesia, más aún cuando se remonta a etapas donde Gutiérrez reiteradamente señala que estamos pasando de una sociedad agrario-urbana a otra urbano-industrial. Remarca que en Europa ese proceso estuvo ligado al secularismo, aunque este solo fue un episodio más coyuntural que estructural. 
Una perspectiva de Derechos Humanos precisa construir una sociedad con justicia social y menos desigualdad económica, considerando esta como elemento concomitante al desarrollo. Julio Cotler señala que "el Perú es uno de los países más desiguales de América Latina, no solo en términos económicos sino también en términos sociales, étnicos, culturales y regionales" (Cotler \& Cuenca, 2011, p. 18).

Por su parte, Contreras afirma que si bien nuestro país muestra niveles de crecimiento sostenido durante los últimos años, reafirma también que el índice Gini de 1980 es exactamente igual que el 2010, de 0,60 (Incio, López, Mazzeo , Mendoza, \& Contreras, 2015), considerado como uno de los más altos del mundo, demostrando así que el crecimiento económico experimentado en las últimas décadas en el país no ha disminuido las brechas de desigualdad que en suma construyen una sociedad sin justicia social.

Esa aproximación de la justicia, los Derechos Humanos y el desarrollo económico podrían constituirse como el elemento articulador, pero somos "conscientes de la necesidad de encontrar balances entre el funcionamiento adecuado de la economía, que requiere disciplina y buena macroeconomía, al mismo tiempo tenemos que cuidar que existan los factores de cohesión social" (Iglesias, 2003, pág. 21); esta sería una aspiración racional de todo académico del presente siglo.

Debemos reconocer que la sociedad peruana se sostiene en una base legal solvente y plenamente fundamentada, el sistema de justicia en el Perú está conformado, en lo medular, por el Poder Judicial, el Consejo Nacional de la Magistratura, el Ministerio de Justicia, la Defensoría del Pueblo, el Ministerio Público, el Tribunal Constitucional $y$ otros organismos que cumplen funciones vinculadas al ámbito jurisdiccional (Miranda Canales, 2007), sin embargo, las implicancias de algunas leyes generan esa invisibilidad de poblaciones que lejos de encontrar protección legal, fomentan su desequilibrio social enclaustrados en la discriminación legal. 


\section{Conclusiones}

La presente investigación demuestra que en sectores donde el contexto social muestra grandes brechas de desigualdad, los factores articuladores legales se convierten en elementos disociadores, que generan flagrantes vulneraciones a los Derechos Humanos. El Decreto Supremo $N^{0}$ 027-2005-AG, crea la reserva paisajista subcuenca del Cotahuasi como Área Natural Protegida, con el objetivo de conservar la diversidad biológica, cultural, paisajística y de ecosistemas; sin embargo no se ha previsto un presupuesto para tal fin y por el contrario se han limitado inversiones en la industria y la minería, lo que resulta una vulneración de los derechos humanos de pobladores que a la fecha no gozan de servicios básicos (luz, agua, desagüe, etc.). A su vez, el Decreto Legislativo 776 referido al fondo de compensación municipal discrimina de manera objetiva a sectores rurales, pues reduce su dinámica de inversión, en detrimento de los derechos de poblaciones pequeñas. La ley de Distribución de canon $\mathrm{N}^{\circ} 27506$ afecta los derechos de poblaciones menores, y favorece a sectores urbanos con servicios integrales. Por lo que planteamos modificación del decreto legislativo 776 del Foncomún y la ley de distribución de canon de manera que la distribución de recursos tenga como prioridad el Índice de Desarrollo Humano y como premisa que son sectores vulnerables con niveles altos de pobreza.

\section{Referencias}

Amaro-Rosales, M., \& De Gortari Rabicla, R. (2016). POLÍTICAS DETRANSFERENCIA TECNOLÓGICA E INNOVACIÓN EN EL SECTOR AGRÍCOLA MEXICANO. Agricultura, Sociedad y Desarrollo, 13(3). Recuperado el 20 de diciembre de 2018

Angles Hernandez, M. (2014). Jurisprudencia interamericana. Acicate contra la discriminación y exclusión de pueblos originarios de México en relación con sus recursos naturales. Anuario Mexicano de Derecho Internacional, 14(1), 261-299. Obtenido de http://www.scielo.org.mx/pdf/amdi/v14/v14a8.pdf

Ansion, J., Mujica , L., Piras, G., \& Villacorta, A. M. (2013). Redes y Maletas: Capital Social en Familias de Migrantes (Primera Edición ed.). (CISEPA, Ed.) Lima, Perú: Pontificia Universidad Católica del Perú . Obtenido de http://wwwpucp.edu.pe/cisepa/ 
BCR. (2016). Informe Economico Regional, Arequipa: Banco Central de Reserva del Perú. Recuperado de http://www.bcrp.gob.pe/publicaciones2/glosario/42transparencia/comunicaciones3/356-informe-economico-y-socialregion-arequipa.html

Campos, X., \& Salas, X. (2016). Gestión planta en Sistemas Agroforestales de Rosetophyllous los bosques en el Valle de TehuacanMexico. Economic Botany, 70(1).

Chigbu, U. E. (2015). Ruralización: una herramienta para la transformación rural. (T. \&. Group, Ed.) Development in Practice, 25(7), 1067-1073. Recuperado el 20 de diciembre de 2018, de http://dx.doi.org/10.1080/09614524.2015.1071783

Instituto Peruano de Economía (2018).Índice de Competitividad Regional. Obtenido de http://www.ipe.org.pe/portal/incore-2018indice-de-competitividad-regional/

Congreso de la República. (1997). Ley Nº 26834 de Areas Naturales Protegidas. Obtenido de http:/www.minam.gob.pe/wpcontent/uploads/2017/04/Ley-N\%C2\%B0-26834.pdf

Congreso de la Republica. (2001). Ley de Canon N²7506,Perú. Obtenido de https://www.mef.gob.pe/es/por-instrumento/ley/6055ley-n-27506/file

Consorcio de Universidades. (2016). Metas del Perú al Bicentenario. Lima. Obtenido de www.consorcio.edu.pe

Cotler, J., \& Cuenca, R. (2011). Las Desigualdades en el Perú, Balances Críticos (Primera Edición ed.). Obtenido de www.iep.org.pe

Daza, H. (2010). La Sociedad Moderna. Revista Venezolana de Economía y Ciencias, 16(2), 61-83. Obtenido de https://www.redalyc.org/pdf/177/17731129004.pdf

ESCALE. (2016). Estadística de la Calidad Educativa. (M. d. Perú, Editor). Obtenido de http://escale.minedu.gob.pe/magnitudes

Guadarrama Gonzales, P. (2015). Derechos humanos y democracia en el pensamiento ilustrado latinoamericano. Revista de Estudios Latinoamericanos, 60(1), 239-280. Recuperado de 
https://www.sciencedirect.com/science/article/pii/S1665857415000 095

Guillen Romo, H. (2005). Obtenido de Francois Perroux: Pionero Olvidado de la Economía del desarrollo. Mundo Siglo XXI. 11(9), 11-22. www.mundosigloxxi.ipn.mx/pdf/v03/11/02.pdf

Gutierrez, G. (1980). La Fuerza Histórica de los Pobres (Segunda Edición ed.). Lima: CEP Centro de Estudios y publicaciones.

Iglesias, E. (2003). El Panorama Actual de los Derechos Humanos y la Democracia. Revista IIDH, 40(1), 13-22. Obtenido de http://www.corteidh.or.cr/tablas/R08064-1.pdf

Incio, J., López, S., Mazzeo , C., Mendoza, W., \& Contreras, C. (2015). La desigualdad de la Distribución de los Ingresos en el Perú (Primera edición ed.). Lima, Perú: Fondo Editorial de la Pontificia Universidad Católica del Perú. Obtenido de www.fondoeditorial.pucp.edu.pe

INEI. (mayo de 2016). Obtenido de Estadisticas de la Calidad Educativa ESCALE. Recuperado de: http://escale.minedu.gob.pe/ueetendencias2016?p_auth $=\mathrm{cP} 906 \mathrm{em}$ W\&p_p_id=TendenciasActualPortlet2016_WAR_tendencias $2016 p$ ortlet_INSTANCE_t6xG\&p_p_lifecycle $=\overline{1} \& \mathrm{p} \_\mathrm{p} \_$state $=$normal $\& \mathrm{p}$ p_mode $=$ view\&p_p_col_id $=$ column-

$1 \& p \_p \_c o l \_p o s=1 \& p \_p \_c o l \_c o u n t=3 \& \_$TendenciasActualPort

INEI. (mayo de 2016). Obtenido de Relación de dependencias policiales. Recuparado de: https://www.inei.gob.pe/descargar/encuestas/.../relacion_dependenc ias_policiales.pdf/

Matijasevic Arcila, M. T., \& Ruiz Silva, A. (2013). La construcción social de lo rural. (ReLMIS, Ed.) Revista Latinoamericana de Metodología de la Investigación Social., 3(5), 24-41. Recuperado el 26 de febrero de 2019, de http://www.relmis.com.ar/ojs/index.php/relmis/article/view/60

Ministerio de Economia y Finanzas. (2004). Portal de transparencia económica. Fondo de Compensación Municipal. Perú. Obtenido de https://www.mef.gob.pe/es/transferencias-a-gobierno-nacionalregional-y-locales/base-legal-y-aspectos-metodologicos/fondo-decompensacion-municipal-foncomun 
Ministerio de Economia y Finanzas. (2017). Presupuestos 2017 FONCOMUN por distritos y provincias. Obtenido de https://www.mef.gob.pe/contenidos/presu_publ/anexos/anexo7_ley 30518.pdf

MINJUS. (2009).Corte Superior de Justicia de Arequipa. Recuperado de: https://www.pj.gob.pe/wps/wcm/connect/CTDP/s_corte_suprema_ utilitarios/as_descarga_procesal/as_equipo_organos_permanentes/a s_arequipa/

MINSA. (2016). Poblacion Estimada por edades simples y grupos de edad, según la proviniia y ditrito departamento de Arequipa - año 2016.

Recuperado de: http://www.minsa.gob.pe/estadisticas/estadisticas/poblacion/poblac ionmarcos.asp?04

Miranda Canales, M. J. (2007). ESTRUCTURA ORGANIZACIONAL PIRAMIDAL DE LOS ORGANOS JURISDICCIONALES EN EL PERU Y EN EL EXTRANJERO. REVISTA OFICIAL DEL PODER JUDICIAL JUSPER. 1(1), 85106. Obtenido https://www.pj.gob.pe/wps/wcm/connect/d183b38046cf1527a502a 544013c2be7/5+Doctrina+Nacional+-+Magistrados++ Miranda+Canales.pdf?MOD=AJPERES\&CACHEID $=\mathrm{d} 183 \mathrm{~b} 3804$ $6 \mathrm{cf} 1527 \mathrm{a} 502 \mathrm{a} 544013 \mathrm{c} 2 \mathrm{be} 7$

PCM. (2012). Sistema Nacional Georeferenciado. Obtenido de http://www.sayhuite.gob.pe/sayhuite/map.phtml

PNUD. (2012). Indice de Desarrollo Humano Departamental. Recuperado de www.pe.undp.org/content/.../pe.Indice $\% 20 \mathrm{de} \% 20$ Desarrollo\%20H umano\%20Perú.xlsx

RIMISP. (2018). Pobreza y Desigualdad Informe Latinoamericano (Primera Edición ed.). (F. Grez, Ed.) Santiago, Chile: Centro Latinoamericano para el Desarrollo rural.

Santos, M. (2014). La discriminación racial, étnica y social en el Perú: balance crítico de la evidencia empírica reciente. Debates.39(1), 5-37. Recuperado de: http://revistas.pucp.edu.pe/index.php/debatesensociologia/article/do wnload/10966/11477/0 
Sen, A. K. (2013). Desarrollo y Libertad (Primera edición ed.). Buenos Aires, Argentina: Planeta.

Serrano, S., \& Vazquez, D. (2011). Fundamentos Teoricos de los Derechos Humanos. (A. N. Cortez, Ed.) México: Comisión de Derechos Humanos del Distrito Federal. Recuperado de: www.cdhdf.org.mx

Sociedad Nacional de Mineria, Petroleo y Energía. (2017). Reporte de Canon. Recuperado de: http://www.snmpe.org.pe/informes-ypublicaciones/canon/canon-minero/4678-reporte-canonminero2017.html

Touraine, A. (1995). ¿Que es el Desarrollo? .Lima: Pontificia Universidad Católica del Perú.

Zegarra Lopez, J. (2015). Propuestas de políticas para los gobiernos regionales 2015-2018. Recuperado de www.cies.org.pe: http://www.cies.org.pe/sites/default/files/investigaciones/009jorge_zegarra.pdf 